\title{
Robust Active MPC Synchronization for Two Discrete-Time Chaotic Systems with Bounded Disturbance
}

\author{
Longge Zhang \\ Department of Mathematics and Physics, North China Electric Power University, Baoding 071003, China \\ Correspondence should be addressed to Longge Zhang; longge@ncepu.edu.cn
}

Received 24 February 2017; Accepted 19 April 2017; Published 30 May 2017

Academic Editor: Qamar Din

Copyright (C) 2017 Longge Zhang. This is an open access article distributed under the Creative Commons Attribution License, which permits unrestricted use, distribution, and reproduction in any medium, provided the original work is properly cited.

\begin{abstract}
This paper proposes a synchronization scheme for two discrete-time chaotic systems with bounded disturbance. By using active control method and imposing some restriction on the error state, the computation of controller's feedback matrix is converted to the min-max optimization problem. The theoretical results are derived with the aid of predictive model predictive paradigm and linear matrix inequality technique. Two example simulations are performed to show the effectiveness of the designed control method.
\end{abstract}

\section{Introduction}

Since the pioneering work of Pecora and Carroll [1], synchronization of chaotic systems has attracted more and more interest due to its significant applications in many fields such as chemical systems [2], ecological systems [3], physical systems [4], and secure communications [5]. From then, many methods have been proposed to study synchronization of chaotic systems. A vast variety of synchronization schemes have been proposed and applied in many research fields, such as adaptive control method [6], feedback control method [7], model predictive control method [8], impulse control method [9], and sliding mode method [10]. However, most of the designed methods have been applied only to investigate continuous-time chaotic systems' synchronization.

In practice, discrete-time chaotic dynamical systems are more important than continuous ones, and many models including neural networks, biological process, physical process, and chemical process are described by discrete-time chaotic dynamical models [11]. Therefore, the research of discretetime chaotic systems' synchronization also plays an important role. Recently, many researches pay more and more attention to the synchronization of discrete-time chaotic systems for its applications in many fields such as secure communication and cryptology, and many synchronization methods are proposed like variable structure control [12],
$H_{\infty}$ control [13], backstepping scheme [14, 15], digital filter method [16], nonlinear control [17], adaptive control [18, 19], active model predictive control [8], and so on [20-25].

However, most of the aforementioned researches consider the ideal condition without disturbance, and it is not real case in practice. The disturbance not only destroys the system's performance but also even makes the system not stable. So it is important to consider the discrete-time chaos synchronization with additive disturbance.

Based on our previous work [8], in this paper we propose a synchronization scheme for two discrete-time chaotic systems with bounded disturbance. The designed method is mainly based on the model predictive control (MPC), and the most difficulty is how to eliminate the influence of the additive disturbance on the stability of closed system. With the aid of quadratic boundedness, it is proved that the designed closed system is stable and realizes the two discrete-time chaotic systems' synchronization.

Notations. $x^{\prime}$ is the transpose vector $x$, and for (semi-)positive-definite matrix $T,\|x\|_{T}=x^{\prime} T x . I$ is the identity matrix. $\varepsilon_{P}:=\left\{\xi: \xi^{\prime} P \xi \leq 1\right\}$ defines the ellipsoid. Co $\{\cdot\}$ denotes a convex combination of the elements in $\{\cdot\}$. The symbol $*$ induces a symmetric structure in linear matrix inequalities. $x(i \mid k)$ is the prediction value of $x$ at time $k+i$. 


\section{Preliminary}

In this section, the definition of quadratic boundedness is revisited [26]. Consider the following discrete-time system described by

$$
x(k+1)=G(k) x(k)+H(k) w(k),
$$

where $x(k) \in R^{n}$ is the state vector and $w(k) \in R^{p}$ is the noise vector. It is assumed that $w(k)$ belongs to an ellipsoidal compact set $\varepsilon_{T}$ with $T>0 . G(k)$ and $H(k)$ are two matrices with appropriated dimension, and suppose that they are unknown but belong to a known bounded set.

Definition 1 (see [26]). A set $\varphi$ is said to be strictly quadratically bounded with a common Lyapunov matrix of system (1) for all $w(k) \in \varepsilon_{T},(G(k), H(k)) \in \varphi, k=0,1, \ldots$, if $x^{\prime}(k) P x(k)>1$ implies $(G x+H w)^{\prime} P(G x+H w)<x^{\prime}(k) P x(k)$, for any $w(k) \in \varepsilon_{T}$ and $(G, H) \in \varphi$.

Definition 2 (see $[26,27]$ ). A set $\varphi$ is said to be a positively invariant set for system (1) for all $w(k) \in \varepsilon_{T},(G(k), H(k)) \in \varphi$, $k=0,1, \ldots$, if $x \in \varphi$ implies $G x+H w \in \varphi$, for any $w(k) \in \varepsilon_{T}$ and $(G, H) \in \varphi$.

\section{Problem Formulation and Controller Design}

Consider the following form of master chaotic system:

$$
x(k+1)=A(k) x(k)+f(x(k), k)+w(k),
$$

where $x \in R^{n}$ is the system's state vector and $A(k) \in \Omega=$ $\operatorname{Co}\left\{A_{1}, A_{2}, \ldots, A_{L}\right\}$ is unknown constant matrix with appropriate dimension. $f(x(k), k)$ is the nonlinear part and $w(k) \in$ $\varepsilon_{P_{w}} \subset R^{n}$ is the bounded disturbance vector. The controlled slave chaotic system is described by

$$
y(k+1)=A(k) y(k)+f(y(k), k)+u(k),
$$

where $y \in R^{n}$ is the slave system's state vector and $u(k)$ is the designed controller.

Define the system error

$$
e(k)=y(k)-x(k) .
$$

Then we can get the error system

$$
\begin{aligned}
e(k+1)= & A(k) e(k)+f(y(k), k)-f(x(k), k) \\
& -w(k)+u(k) .
\end{aligned}
$$

With the aid of the active control technique [8, 28, 29], we select the following controller:

$$
\begin{aligned}
u(k)=u_{1}(k)-f(y(k), k)+f(x(k), k), & \\
& u_{1}(k)=F(k) x(k),
\end{aligned}
$$

where $F(k)$ will be computed in the following optimization problem. Substituting (6) into (5), we can get

$$
e(k+1)=[A(k)+F(k)] e(k)-w(k) ;
$$

that is,

$$
e(k+1)=\sum_{l=1}^{L} w_{l}(k)\left[A_{l}+F(k)\right] e(k)-w(k) .
$$

Lemma 3 (see [26]). For system (8), the following facts are equivalent:

(a) For all allowable $w(k) \in \varepsilon_{P_{w}}, k \geq 0$, (8) is strictly quadratically bounded with a common Lyapunov matrix $M>0$.

(b) For any $w(k) \in \varepsilon_{P_{w}}$ and any $l \in\{1, \ldots, L\}, e^{T} M e>1$ implies

$$
\begin{aligned}
& {\left[\left(A_{l}+F\right) e-w\right]^{T} M\left[\left(A_{l}+F\right) e-w\right]<e^{T} M e} \\
& \text { and } e^{T} M e=1 \text { implies } \\
& {\left[\left(A_{l}+F\right) e-w\right]^{T} M\left[\left(A_{l}+F\right) e-w\right] \leq e^{T} M e .}
\end{aligned}
$$

(c) For any allowable $w(k) \in \varepsilon_{P_{w}}, k \geq 0$, the ellipsoid $\varepsilon_{M}$ is a positively invariant set for (8).

(d) There exists $\alpha \in(0,1)$, such that

$$
\left[\begin{array}{ccc}
(1-\alpha) M & * & * \\
0 & \alpha P_{w} & * \\
M\left(A_{l}+F\right) & -M & M
\end{array}\right] \geq 0, \quad l \in\{1, \ldots, L\}
$$

The target of the following part is to solve a dynamic feedback MPC where, at each time $k$, the feedback matrix is computed through the following optimization problem:

$$
\min _{\beta<1, \gamma, Q, F} \max _{[A] \in \Omega, w(k+i) \in \varepsilon_{P_{w}}, i=0,1, \ldots, N-1} J_{N}(k),
$$

where

$$
J_{N}(k)=\sum_{i=0}^{N}\|e(i \mid k)\|_{R}
$$

and $R$ is a symmetric positive-definite weighting matrix. Consider a quadratic Lyapunov function $V(e)=e^{i} P e(P>0)$ of the error state $e$ with $V(0)=0$. At every sampling time $k$, suppose the error state $e$ and $V$ satisfy the following condition:

$$
\begin{gathered}
e(k) \in \varepsilon_{Q^{-1}} \\
e\left(i_{1} \mid k\right) \in \varepsilon_{Q^{-1}} \backslash \varepsilon_{\beta^{-1} Q^{-1}} \\
0 \leq i_{1}<N \\
e\left(i_{2} \mid k\right) \in \varepsilon_{\beta^{-1} Q^{-1}}, \quad \forall i_{2} \geq N \\
\|e(i \mid k)\|_{Q^{-1}}^{2} \geq 1 \Longrightarrow \\
\|e(i \mid k)\|_{Q^{-1}-\|e(i+1 \mid k)\|_{Q^{-1}}^{2}}^{2} \geq \frac{1}{\gamma}\|e(i \mid k)\|_{R}^{2},
\end{gathered}
$$


where $Q^{-1}=P$. It is obvious that condition (14) consists of the initial condition of the min-max problem, (15) implies $e(k)$ within $\varepsilon_{\mathrm{Q}^{-1}}$, (15) guarantees that system (7) is quadratically bounded with a common Lyapunov matrix $Q^{-1}$, and (16) is used to guarantee convergence of $e(k)$ towards $\varepsilon_{\beta^{-1} Q^{-1}}$ when $e(k) \notin \varepsilon_{Q^{-1}}$. N need not to be known and $\beta<1$ is to ensure that $e(k) \notin \varepsilon_{\beta^{-1} Q^{-1}}$. Note that, since $e(k)$ can only convergence to a neighborhood of $e(k)=0$, there exist $k>0$ when (16) becomes infeasible. In this case, we will stop computing the optimization problem.

Since $w(k) \in \varepsilon_{P_{w}}, e(k) \notin \varepsilon_{\beta^{-1} Q^{-1}}$ is equivalent to

$$
\begin{aligned}
\beta\|w(k+i)\|_{P_{w}}^{2} & \leq\|e(i \mid k)\|_{Q^{-1}}^{2} \Longrightarrow \\
\|e(i \mid k)\|_{Q^{-1}}^{2}-\|e(i+1 \mid k)\|_{Q^{-1}}^{2} & \geq \frac{1}{\gamma}\|e(i \mid k)\|_{R} .
\end{aligned}
$$

By applying (8) and using the S-procedure [30], we can get that the result of (18) is satisfied if and only if

$$
\begin{aligned}
\exists \alpha \geq 0 & :\left[\begin{array}{cc}
\gamma Q^{-1}-\gamma[A+F]^{T} Q^{-1}[A+F] & * \\
\gamma Q^{-1}[A+F] & -\gamma Q^{-1}
\end{array}\right] \\
& -\alpha \gamma\left[\begin{array}{cc}
Q^{-1} & 0 \\
0 & -\beta P_{w}
\end{array}\right] \geq 0 .
\end{aligned}
$$

Using Schur complement it is shown that (19) is guaranteed by

$$
\left[\begin{array}{ccccc}
(1-\alpha) Q^{-1} & * & * & * & * \\
0 & \alpha \beta P_{w} & * & * & * \\
-Q^{-1}\left[A_{l}+F\right] & Q^{-1} & Q^{-1} & * & * \\
R^{1 / 2} & 0 & 0 & \gamma I & * \\
0 & 0 & 0 & 0 & I
\end{array}\right] \geq 0
$$

$$
l=1, \ldots, L .
$$

By pre- and postmultiplying both sides of (20) with $\operatorname{diag}\{\mathrm{Q}, I$, $Q, I, I\}$, then one can obtain

$$
\left[\begin{array}{ccccc}
(1-\alpha) Q & * & * & * & * \\
0 & \alpha \beta P_{w} & * & * & * \\
-\left[A_{l}+F\right] & I & Q & * & * \\
Q R^{1 / 2} & 0 & 0 & \gamma I & * \\
0 & 0 & 0 & 0 & I
\end{array}\right] \geq 0 \quad l=1, \ldots, L .
$$

Summing (17) from $i=0$ to $i=N-1$ and applying (14) yield

$$
J_{N}(k) \leq \gamma\|e(k)\|_{Q^{-1}}^{2} \leq \gamma .
$$

Hence, (18) is imposed not only for quadratic boundedness but also for optimality ( $\gamma$ will be minimized). Then we can get the following theorem.

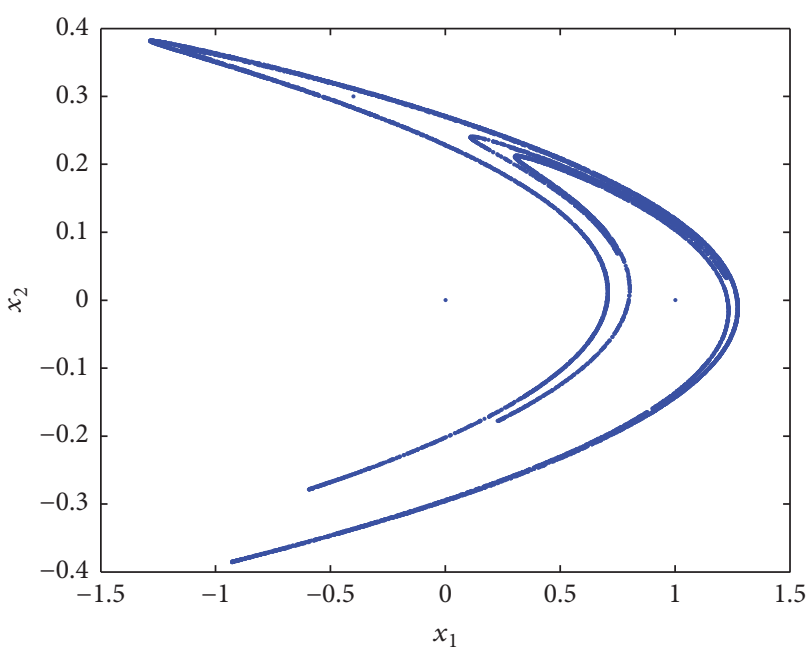

Figure 1: Attractor of Hénon map.

Theorem 4. The master system (2) and slave system (3) can realize the synchronization with controller (6), and the feedback matrix $F$ is computed through the following optimization:

$$
\min _{\beta<1, \gamma, Q, F}
$$

subject to

$$
\begin{aligned}
& {\left[\begin{array}{cc}
1 & * \\
e(k) & Q
\end{array}\right] \geq 0} \\
& {\left[\begin{array}{ccccc}
(1-\alpha) Q & * & * & * & * \\
0 & \alpha \beta P_{w} & * & * & * \\
-\left[A_{l}+F\right] & I & Q & * & * \\
Q R^{1 / 2} & 0 & 0 & \gamma I & * \\
0 & 0 & 0 & 0 & I
\end{array}\right] \geq 0 \quad l=1, \ldots, L .}
\end{aligned}
$$

\section{Numerical Examples}

Example 1. This example verifies the effectiveness of the prosed robust active MPC control method in solving the synchronization between two identical discrete chaotic systems. Consider the well-known discrete Hénon map [31]:

$$
\begin{aligned}
& x_{1}(k+1)=x_{2}(k)+1-a x_{2}(k) \\
& x_{2}(k+1)=b x_{1}(k) .
\end{aligned}
$$

Corresponding to (2), $A=\left(\begin{array}{ll}0 & 1 \\ b & 0\end{array}\right), f(x(k))=\left(\begin{array}{c}1-a x_{2}(k)^{2} \\ 0\end{array}\right)$. The system has a strange attractor when $a=1.4, b=0.3$, and its attractor is shown if Figure 1 with the initial value $x(0)=$ $(0,0)^{T}$. The master system is selected as

$$
\begin{aligned}
& x_{1}(k+1)=x_{2}(k)+1-a x_{2}(k)+0.25 \sin (k) \\
& x_{2}(k+1)=b x_{1}(k)+0.25 \cos (k) .
\end{aligned}
$$


When parameter $b$ varied between 0.2 and 0.4 , the matrix $A$ belongs to the polytope $\Omega=\operatorname{Co}\left\{A_{1}, A_{2}\right\}$, where $A_{1}=\left(\begin{array}{cc}0 & 1 \\ 0.2 & 0\end{array}\right)$ and $A_{2}=\left(\begin{array}{cc}0 & 1 \\ 0.4 & 0\end{array}\right)$. And the slave system is chosen as

$$
y(k+1)=A y(k)+f(y(k), k)+u(k),
$$

where $y=\left(y_{1}, y_{2}\right)^{T}$ is the slave system's state and it is obvious that $P_{w}=4 I$. When we select $\alpha=0.5, \beta=0.9$, and $R=2 I$, and the initial values are selected as $x(0)=(16,-8)^{T}, y(0)=$ $(2,6)^{T}$, then the error of the master system and slave system is shown in Figure 2 with controller (6).

Example 2. In this example, the proposed robust active MPC controller is applied to stabilize the uncertain generalized hyperchaotic Hénon map [32]:

$$
\begin{aligned}
& x_{1}(k+1)=1+x_{3}(k)-a x_{2}(k)^{2} \\
& x_{2}(k+1)=1+b x_{2}(k)-a x_{1}(k)^{2} \\
& x_{3}(k+1)=b x_{1}(k) .
\end{aligned}
$$

When $a=1.4, b=0.2$ the generalized Hénon map has an attractor which is shown in Figure 3 with the initial values $x(0)=(0.1,0.05,0.3)^{T}$. The master system is selected as

$$
\begin{aligned}
& x_{1}(k+1)=1+x_{3}(k)-a x_{2}(k)^{2}+0.25 \sin (k) \\
& x_{2}(k+1)=1+b x_{2}(k)-a x_{1}(k)^{2}+0.25 \cos (k) \\
& x_{3}(k+1)=b x_{1}(k) 0.15 \sin (2 k) .
\end{aligned}
$$

When parameter $b$ varied between 0.1 and 0.3 , the matrix $A$ belongs to the polytope $\Omega=\operatorname{Co}\left\{A_{1}, A_{2}\right\}$, where $A_{1}=$ $\left(\begin{array}{ccc}0 & 0 & 1 \\ 0 & 0.1 & 0 \\ 0.1 & 0 & 0\end{array}\right)$ and $A_{2}=\left(\begin{array}{ccc}0 & 0 & 1 \\ 0 & 0.3 & 0 \\ 0.3 & 0 & 0\end{array}\right)$. The slave system is selected

$$
y(k+1)=A y(k)+g(y(k), k)+u(k),
$$

where $y(k)=\left(y_{1}(k), y_{2}(k), y_{3}(k)\right)^{T}, A=\left(\begin{array}{lll}0 & 0 & 1 \\ 0 & b & 0 \\ b & 0 & 0\end{array}\right)$, $f(y(k))=\left(\begin{array}{c}1-a y_{2}(k)^{2} \\ 1-a y_{1}(k)^{2} \\ 0\end{array}\right)$.

When solving the optimization (23), we select the parameters as $P_{w}=4 I, \alpha=0.5, \beta=0.9$, and $R=I$ and the simulation error of the master system and the slave system is shown in Figure 4 with the initial value of $x(0)=(16,-8,1)^{T}$ and $y(0)=(0,4,-5)^{T}$.

\section{Conclusions}

In this paper, the robust active MPC synchronization for two discrete-time chaotic systems with bounded disturbance is studied. With the help of the active control technique, the synchronization problem is changed into a min-max optimization problem. On the basis of discrete Lyapunov stability theory, we prove the stability of the closed-loop system. Two simulation examples demonstrate that the designed control technique can synchronize the two uncertain discrete-time chaotic systems.
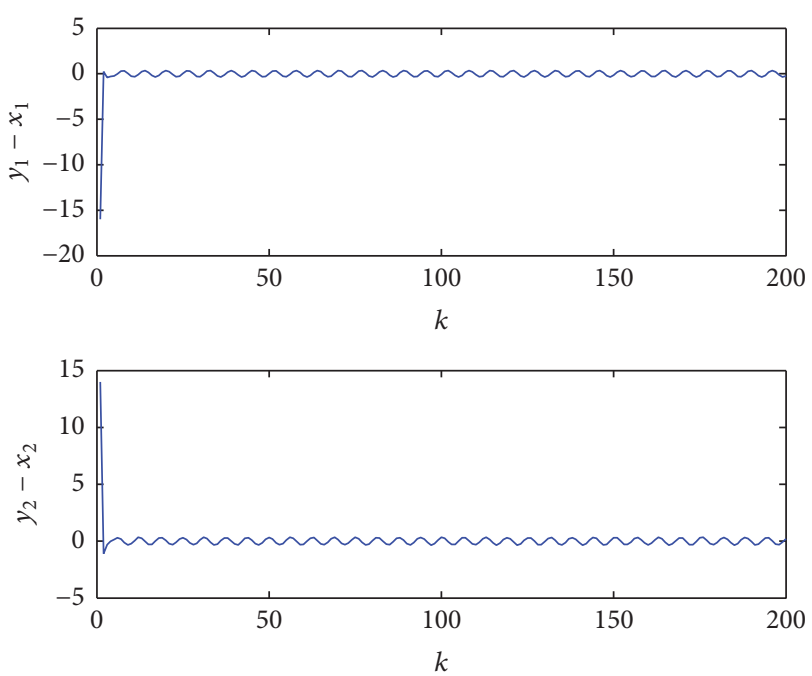

FIGURE 2: Synchronization errors between the uncertain disturbance Hénon maps.

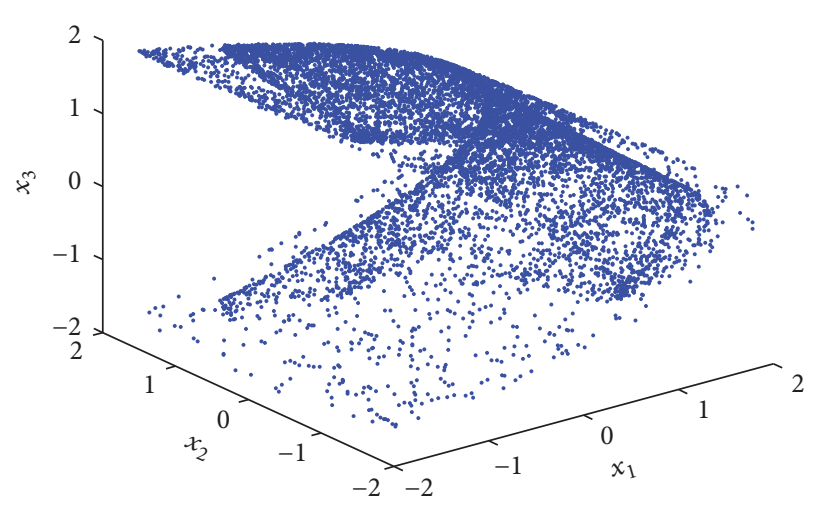

Figure 3: Attractor of the generalized Hénon map.
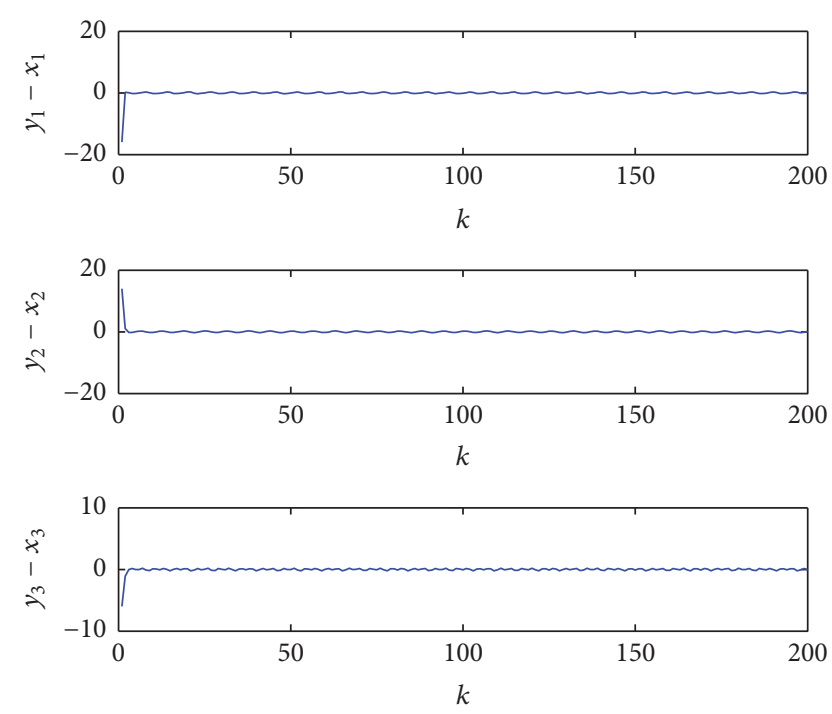

FIGURE 4: Synchronization errors between the generalized Hénon maps. 


\section{Conflicts of Interest}

The author declares no conflicts of interest.

\section{Acknowledgments}

This research was supported by the National Natural Science Foundation of Hebei Province under Project no. F2016502025; the Fundamental Research Funds for the Central Universities under Project no. 9161015007; and the National Natural Science Foundation of China under Projects nos. 61273144, 61403137, and 61603134 .

\section{References}

[1] L. M. Pecora and T. L. Carroll, "Synchronization in chaotic systems," Physical Review Letters, vol. 64, no. 8, pp. 821-824, 1990.

[2] Y.-N. Li et al., "Experimental study of chaos synchronization in the Belousov-Zhabotinsky chemical system," Chaos, Solitons and Fractals, vol. 22, no. 4, pp. 767-771, 2004.

[3] B. Blasius and L. Stone, "Chaos and phase synchronization in ecological systems," International Journal of Bifurcation and Chaos, vol. 10, no. 10, pp. 2361-2380, 2000.

[4] S. Sivaprakasam et al., "Experimental demonstration of anticipating synchronization in chaotic semiconductor lasers with optical feedback," Physical Review Letters, vol. 87, no. 15, Article ID 154101, p. 154101, 2001.

[5] L.-f. Zhang, X.-l. An, and J.-g. Zhang, "A new chaos synchronization scheme and its application to secure communication," Nonlinear Dynamics. An International Journal of Nonlinear Dynamics and Chaos in Engineering Systems, vol. 73, no. 1-2, pp. 705-722, 2013.

[6] S. Chen and J. Lü, "Synchronization of an uncertain unified chaotic system via adaptive control," Chaos, Solitons and Fractals, vol. 14, no. 4, pp. 643-647, 2002.

[7] M. Rafikov and J. M. Balthazar, "On control and synchronization in chaotic and hyperchaotic systems via linear feedback control," Communications in Nonlinear Science and Numerical Simulation, vol. 13, no. 7, pp. 1246-1255, 2008.

[8] L. Zhang and X. Liu, "The synchronization between two discrete-time chaotic systems using active robust model predictive control," Nonlinear Dynamics. An International Journal of Nonlinear Dynamics and Chaos in Engineering Systems, vol. 74, no. 4, pp. 905-910, 2013.

[9] X. Li and S. Song, "Research on synchronization of chaotic delayed neural networks with stochastic perturbation using impulsive control method," Communications in Nonlinear Science and Numerical Simulation, vol. 19, no. 10, pp. 3892-3900, 2014.

[10] S. Vaidyanathan, "Global chaos synchronisation of identical LiWu chaotic systems via sliding mode control," International Journal of Modelling, Identification and Control, vol. 22, no. 2, pp. 170-177, 2014.

[11] A. Ouannas, Z. Odibat, and N. Shawagfeh, "A New Q-S Synchronization Results for Discrete Chaotic Systems," Differential Equations and Dynamical Systems, pp. 1-10, 2016.

[12] T.-L. Liao and N.-S. Huang, "Control and synchronization of discrete-time chaotic systems via variable structure control technique," Physics Letters. A, vol. 234, no. 4, pp. 262-268, 1997.

[13] D. Qi, M. Liu, M. Qiu, and S. Zhang, "Exponential $H_{\infty}$ synchronization of general discrete-time chaotic neural networks with or without time delays," IEEE Transactions on Neural Networks, vol. 21, no. 8, pp. 1358-1365, 2010.
[14] Z. Yan, "Q-S (complete or anticipated) synchronization backstepping scheme in a class of discrete-time chaotic (hyperchaotic) systems: a symbolic-numeric computation approach," Chaos. An Interdisciplinary Journal of Nonlinear Science, vol. 16, no. 1, Article ID 013119, 013119, 11 pages, 2006.

[15] L. Huang, M. Wang, and R. Feng, "Synchronization of generalized Henon map via backstepping design," Chaos, Solitons and Fractals, vol. 23, no. 2, pp. 617-620, 2005.

[16] M. Eisencraft, R. D. Fanganiello, and L. A. Baccala, "Synchronization of discrete-time chaotic systems in bandlimited channels," Mathematical Problems in Engineering, vol. 2009, Article ID 207971, 2009.

[17] Z. Yan, "A nonlinear control scheme to anticipated and complete synchronization in discrete-time chaotic (hyperchaotic) systems," Physics Letters, Section A: General, Atomic and Solid State Physics, vol. 343, no. 6, pp. 423-431, 2005.

[18] Y. Li, Y. Chen, and B. Li, "Adaptive control and function projective synchronization in 2D discrete-time chaotic systems," Communications in Theoretical Physics, vol. 51, no. 2, pp. 270-278, 2009.

[19] G. Feng and G. Chen, "Adaptive control of discrete-time chaotic systems: a fuzzy control approach," Chaos, Solitons and Fractals, vol. 23, no. 2, pp. 459-467, 2005.

[20] Y. Gao, X. Zhang, G. Lu, and Y. Zheng, "Impulsive synchronization of discrete-time chaotic systems under communication constraints," Communications in Nonlinear Science and Numerical Simulation, vol. 16, no. 3, pp. 1580-1588, 2011.

[21] L. Zhang and H. Jiang, "Impulsive generalized synchronization for a class of nonlinear discrete chaotic systems," Communications in Nonlinear Science and Numerical Simulation, vol. 16, no. 4, pp. 2027-2032, 2011.

[22] Z. Yan, "Q-S synchronization in 3D Hénon-like map and generalized Hénon map via a scalar controller," Physics Letters A, vol. 342, no. 4, pp. 309-317, 2005.

[23] O. M. Kwon, J. W. Son, and S. M. Lee, "Constrained predictive synchronization of discrete-time chaotic Lur'e systems with time-varying delayed feedback control," Nonlinear Dynamics. An International Journal of Nonlinear Dynamics and Chaos in Engineering Systems, vol. 72, no. 1-2, pp. 129-140, 2013.

[24] Q. Din, "Neimark-Sacker bifurcation and chaos control in Hassell-Varley model," Journal of Difference Equations and Applications, pp. 1-22, 2017.

[25] H. Khalil and R. A. Khan, "A new method based on Legendre polynomials for solutions of the fractional two-dimensional heat conduction equation," Computers \& Mathematics with Applications, vol. 67, no. 10, pp. 1938-1953, 2014.

[26] A. Alessandri, M. Baglietto, and G. Battistelli, "On estimation error bounds for receding-horizon filters using quadratic boundedness," Institute of Electrical and Electronics Engineers. Transactions on Automatic Control, vol. 49, no. 8, pp. 1350-1355, 2004.

[27] B. Ding and L. Xie, "Robust model predictive control via dynamic output feedback," in Proceedings of 7th World Congress on Intelligent Control and Automation, WCICA'08, pp. 3388-3393, chn, June 2008.

[28] H. N. Agiza and M. T. Yassen, "Synchronization of Rossler and Chen chaotic dynamical systems using active control," Physics Letters. A, vol. 278, no. 4, pp. 191-197, 2001.

[29] S. K. Agrawal, M. Srivastava, and S. Das, "Synchronization of fractional order chaotic systems using active control method," Chaos, Solitons \& Fractals, vol. 45, no. 6, pp. 737-752, 2012. 
[30] K. s. Derinkuyu and M. Ç. Pınar, "On the S-procedure and some variants," Mathematical Methods of Operations Research, vol. 64, no. 1, pp. 55-77, 2006.

[31] M. Hénon, "A two-dimensional mapping with a strange attractor," in The Theory of Chaotic Attractors, pp. 94-102, Springer, 1976.

[32] K. Stefański, "Modelling Chaos and Hyperchaos with 3-D Maps," Chaos, Solitons and Fractals, vol. 9, no. 1, pp. 83-93, 1998. 


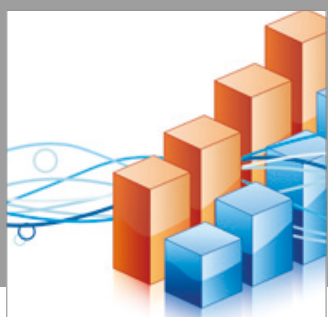

Advances in

Operations Research

vatersals

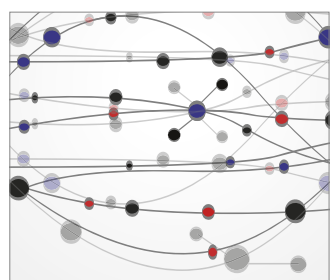

\section{The Scientific} World Journal
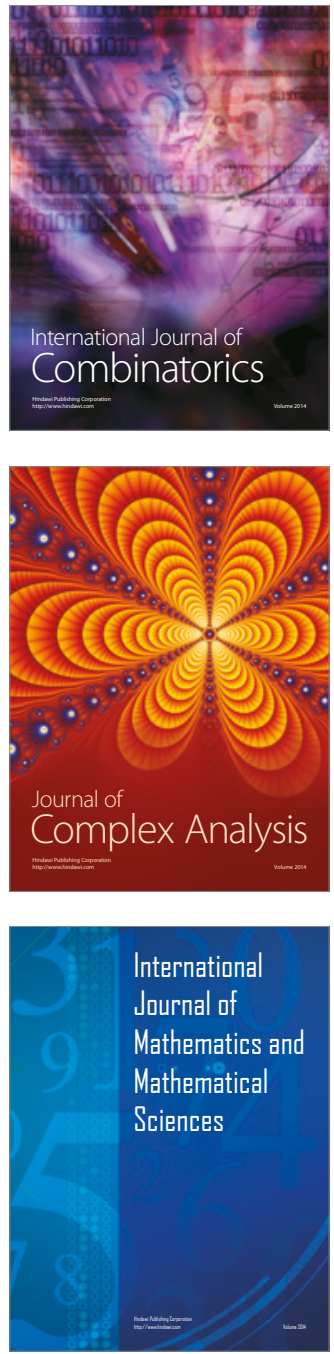
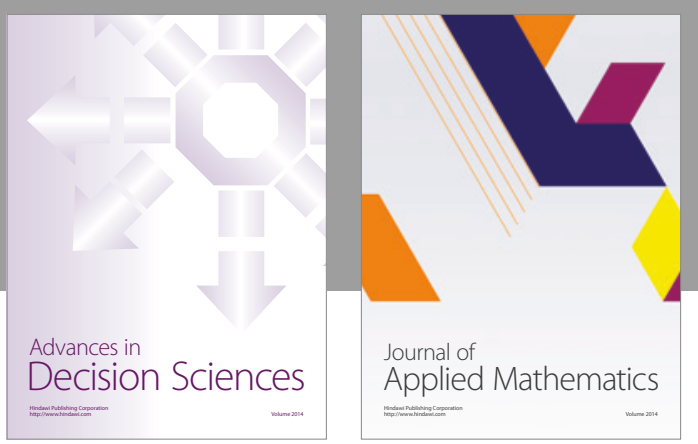

Algebra

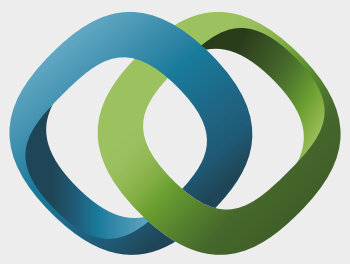

\section{Hindawi}

Submit your manuscripts at

https://www.hindawi.com
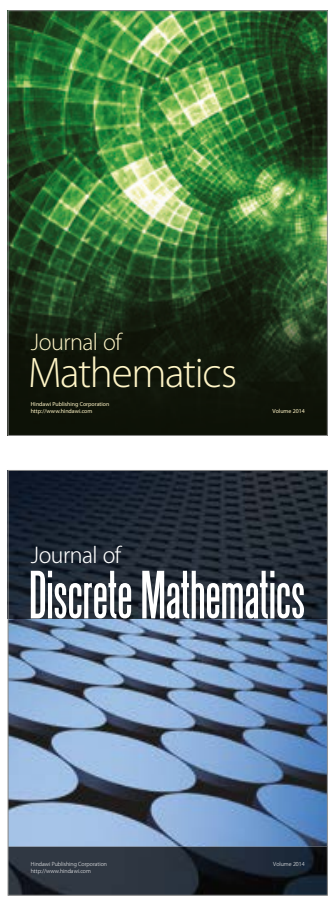

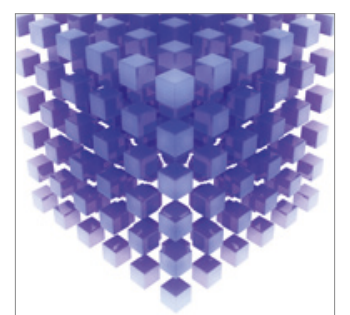

Mathematical Problems in Engineering
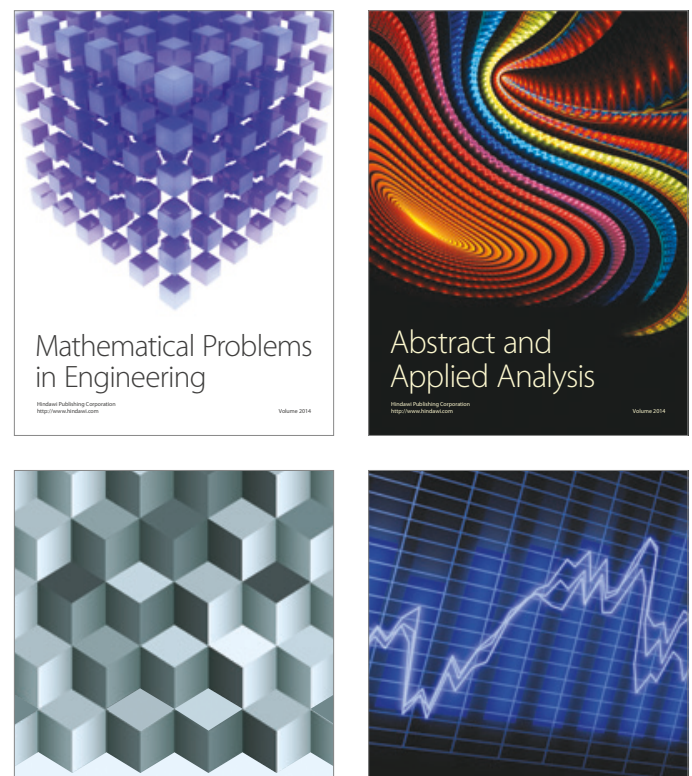

Journal of

Function Spaces

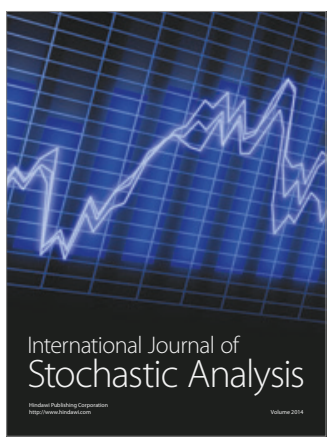

Probability and Statistics
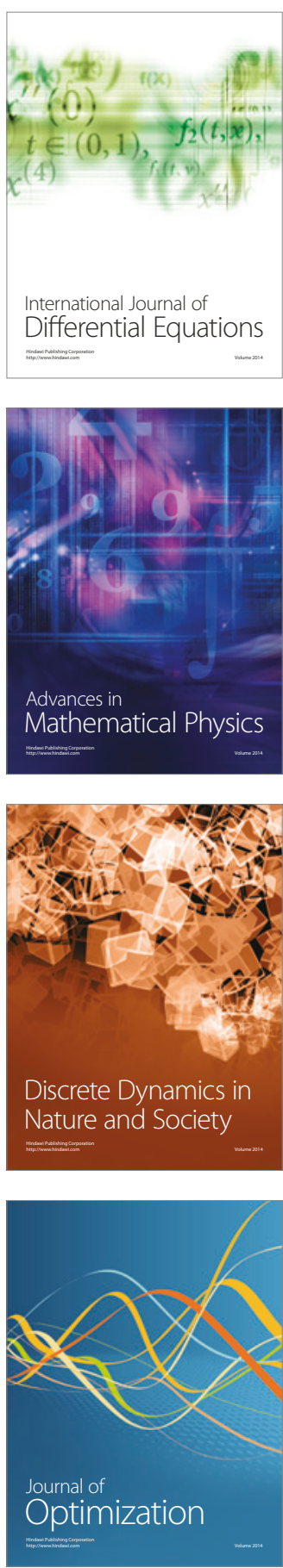